\title{
The origin of cooperativity in high-spin-low-spin transitions in molecular crystals
}

\author{
Andrei L. Tchougreeff ${ }^{1}$ \\ Laboratoire de Chimie Inorganique, URA CNRS 420, Université de Paris-Sud, 91405 Orsay, France
}

Received 17 June 1993; in final form 3 August 1993

\begin{abstract}
A new model for the first-order transitions between the low-spin and high-spin phases of molecular crystals of some transition metal complexes is proposed. The abrupt spin transitions (or those exhibiting thermal hysteresis) are attributed to first-order transitions between the different crystal phases with the sublattice order. The necessary conditions for these transitions to take place are formulated in terms of intramolecular parameters of the spin transition and intermolecular interactions.
\end{abstract}

\section{Introduction}

The transitions between low-spin low-temperature states and high-spin high-temperature states, observed in a series of transition metal complexes (TMC), have attracted attention during the last two decades (for recent reviews see refs. [1,2]). The spin transitions in iron(II) TMC are probably the most extensively studied and they may exhibit a cooperative character.

The theoretical description of the spin transition is based on the model proposed by Slichter and Drickamer (SD) [3,4] which includes intermolecular interactions phenomenologically. This approach, however, faces serious problems, since it requires rather strong interactions favoring the formation of pairs of molecules of the same spin in order to model the cooperativity effects observed experimentally. However, no convincing reasons explaining why the average attraction between isomers of the same spin should be stronger than that of different spins have ever been proposed. It is most likely that the interaction has the opposite sign to that required by the SD model (see below) and thus the approach [3,4] generally does not apply to the analysis of spin transitions in molecular crystals. Here we

1 Permanent address: L.Y. Karpov Institute of Physical Chemistry, ul. Obukha 10, 103064 Moscow, Russian Federation. propose an alternative model for the cooperative spin transitions based on the idea of sublattice order. This model also manifests a first-order phase transition but seems to be free from the shortcomings of the SD model.

\section{Spin transitions and interaction parameters}

The spin transitions are characterized experimentally by the molar fraction $x$ of molecules in the highspin state. The $x(T)$ curves measured in dilute solutions (both liquid and solid) are smooth. For a series of $\mathrm{Fe}$ (II) complexes with bulky organic ligands the $x(T)$ curves in the crystal phase may be either smooth or abrupt or even exhibit a hysteresis, i.e. the transition between the spin states happens at different temperatures upon cooling and upon heating. These features of the $x(T)$ curves together with the specific heat measurements [5] demonstrate that abrupt spin transitions are first-order phase transitions.

SD $[3,4]$ applied the regular solution model (see, for example, refs. [6-8]) to the particular case of the spin transitions in solids. According to refs. $[3,4]$ the free energy of the crystal undergoing the spin transition is given by

$g=(\Delta h-T \Delta s) x+\Gamma x(1-x)+T s_{\operatorname{mix}}$, 
where the mixing entropy is given by

$s_{\text {mix }}=k_{\mathrm{B}}[x \ln x+(1-x) \ln (1-x)]$,

$\Delta h$ and $\Delta s$ are the enthalpy and the entropy differences between the high- and the low-spin isomers. According to refs. [6-8] (see also ref. [9] for the discussion of this problem in the specific context of spin transitions) the phenomenological parameter $\Gamma$ is directly related to the microscopic interaction energies of the spin isomers occupying the neighbour sites,

$\Gamma=\frac{1}{2} z \epsilon, \quad \epsilon=E_{\mathrm{HL}}-\frac{1}{2}\left(E_{\mathrm{LL}}+E_{\mathrm{HH}}\right)$,

where $z$ is the number of neighbours of any given molecule; $E_{\mathrm{HL}}, E_{\mathrm{LL}}$ and $E_{\mathrm{HH}}$ are the energies of the intermolecular interaction in the respective pairs of spin isomers.

The analysis of eq. (1) led SD [3,4] to the conclusion that the spin transition is of the first order when $\Gamma$ is greater than $2 k_{\mathrm{B}} T_{\mathrm{c}}\left(T_{\mathrm{c}}=\Delta h / \Delta s\right)$. The interaction thus must be positive and of the order of several hundreds of kelvin. Much effort has been devoted in order to find out what kind of intermolecular interactions would be capable of giving such large $\Gamma$ values [10-17]. However, no convincing origin of the cooperative (in terms of ref. [9]) interaction has ever been proposed. The spin-vibration (or JahnTeller) interaction [10-12] which is very important as the intimate mechanism of the spin transition on the level of separate molecules does not treat properly the intermolecular coupling.

An effective interaction due to the image pressure between spin active molecules treated as defects embedded in an elastic medium has been proposed in refs. [13-17]. It seems, however, that this approach is not valid in the case of a high concentration of spin active molecules (when the cooperative effects only take place). In this case the only elastic medium is the crystal itself formed by the molecules undergoing the transition. The formulae proposed in refs. [13-17] obviously become invalid when the total volume occupied by defects is comparable to the volume of the crystal [18].

To summarize, we do not see any reliable mechanism which could provide the positive value of $\epsilon$ as required by the SD model. The reason for this obvious failure is that the interaction parameter $\epsilon$ is most likely to be negative. Indeed, TMC with bulky organic ligands capable of undergoing spin transitions are held in the crystals by van der Waals interactions. For describing the intermolecular forces the TMC molecules can be viewed as spheres whose interaction depends on the distance $r$ between their surfaces. In the harmonic approximation the interaction energy for two low-spin molecules is: $E_{\mathrm{LL}}+\frac{1}{2} k(r-d)^{2}$ ( $d$ is the equilibrium distance between the spheric surfaces). The equilibrium distance between the centers of two low-spin isomers of radius $R_{\mathrm{L}}$ is $a_{\mathrm{L}}=2 R_{\mathrm{L}}+d$. When the transition happens the distance between the iron atom and the donor nitrogen atoms of the ligands increases. For the surrounding molecules the transition results in a corresponding increase $\delta R_{\mathrm{HL}}$ of the radius of the sphere. The interaction energies in the pairs of molecules with the centers fixed at the distance $a_{\mathrm{L}}$ now become

$E_{\mathrm{HL}}=E_{\mathrm{LL}}+\frac{1}{2} k \delta R_{\mathrm{HL}}^{2}$,

$E_{\mathrm{HH}}=E_{\mathrm{LL}}+2 k \delta R_{\mathrm{HL}}^{2}$.

Substituting these values in eq. (2) affords,

$\epsilon=-\frac{1}{2} k \delta R_{\mathrm{HL}}^{2}<0$.

Within the proposed model the microscopic elasticity parameter $k$ can be related to the bulk modulus $K: 3 K a_{\mathrm{L}}=k$. The variation of $a$ upon the transition $\Delta a=2 \delta R_{\mathrm{HL}}$ and $\Delta V / V=3 \Delta a / a$, so finally we have a relation between $\epsilon$ and the molecular crystal parameters,

$\epsilon=-K V(\Delta V / V)^{2} / 24 Z$,

where $V$ is the unit cell volume, $\Delta V$ is the volume effect of the transition, and $Z$ is the number of molecules in the unit cell. Substituting the available data $[1,19]$ on spin transitions and the elastic properties of the corresponding crystals results in an $\epsilon$ value of $-60 \mathrm{~K}$ for the [ $\mathrm{Fe}$ (bipy) ${ }_{2}(\mathrm{NCS})_{2}$ ] compound. This value (when multiplied by the proper number of nearest neighbours) gives the correct order of magnitude for the interaction constant $\Gamma$, but with the opposite sign.

The above result has probably been known for a long time, but it obviously cannot be reconciled with the requirements imposed by the SD model. This difficulty cannot be overcome within its framework since the experimentally observed first-order transitions are possible in the model $[3,4]$ (eq. (1)) only 
if the interaction parameter $\Gamma($ or $\epsilon)$ is positive. The interactions with negative values of $\Gamma$ have been called anticooperative [20] and are thought to lead to smoother $x(T)$ curves than those obtained in the dilute solutions. In section 3 we shall construct a theory of the cooperative spin transitions for negative $\epsilon$ values.

\section{Sublattice order and spin transitions}

Let us consider the TMC crystal undergoing the spin transition as a mixture of two spin isomers. It is known (see, for example, ref. [6]) that binary mixtures may undergo sublattice ordering transitions when $\epsilon$ is negative. We assume that the crystal lattice can be divided into two sublattices ( $A$ and $B$ ). The ratio of the numbers of sites in the sublattices $s=N_{\mathrm{B}} / N_{\mathrm{A}}>1$. Following ref. [21] we define the order parameter $\theta$ which describes the excess of the high-spin isomer in the sublattice $A$ with respect to its average fraction $x$,

$x_{\mathrm{A}}=x(1+s \theta), \quad x_{\mathrm{B}}=x(1-\theta)$.

The free energy of the crystal is then given by

$g(T, x, \theta)=g_{0}(T, x)+\Delta g(T, x, \theta)$,

where

$$
\begin{aligned}
& g_{0}(T, x)=(\Delta h-T \Delta s) x \\
& \quad+k_{\mathrm{B}} T[x \ln x+(1-x) \ln (1-x)] \\
& \quad-\frac{1}{2} z|\epsilon| x(1-x)
\end{aligned}
$$

is the regular solution free energy eq. (1), but with the negative interaction parameter $\epsilon=-|\epsilon|$ and the term

$$
\begin{aligned}
& \Delta g(T, x, \theta)=k_{\mathrm{B}} T(1+s)^{-1}\{x(1+s \theta) \ln (1+s \theta) \\
& \quad+(1-x)(1-y s \theta) \ln (1-y s \theta) \\
& \quad+s[x(1-\theta) \ln (1-\theta) \\
& \quad+(1-x)(1+y \theta) \ln (1+y \theta)]\} \\
& \quad-\frac{1}{2} s z z_{\mathrm{eff}}|\epsilon| x^{2} \theta^{2},
\end{aligned}
$$

where $y=x /(1-x)$, describes the sublattice order. ( $z_{\text {eff }}$ is the number of neighbours of a given site, which are situated in the different sublattices divided by the total number of neighbours $z$.)
The regular solution free energy $g_{0}\left(T_{c}, x\right)$ has a unique minimum $x=\frac{1}{2}[3,4,6-8]$. After introducing the new variable $x=\frac{1}{2}+\xi$ one can see that at $T=T_{\mathrm{c}}$ the total free energy eq. (5) remains unchanged under the transformation

$$
\begin{gathered}
\xi^{\prime}=-\xi, \quad \theta^{\prime}=-y \theta \\
\left(y^{\prime}=y^{-1}, \text { etc. }\right) .
\end{gathered}
$$

The point $\theta=0$ (corresponding to the disordered phase of the crystal characterized by the random distribution of the high- and low-spin isomers in the lattice) is always a stationary point of $\Delta g(T, \xi, \theta)$ (minimum or a saddle). If the temperature is low enough $T<T_{0}=z z_{\text {eff }}|\epsilon| / 4 k_{\mathrm{B}}$ the disordered phase becomes unstable and the free energy eq. (5) has a minimum $\left(\xi_{e}, \theta_{\mathrm{e}} \neq 0\right.$ ), corresponding to the sublattice order. It can be shown numerically and also by analysis of the Landau expansion [21] of $g(T, \xi, \theta)$ that $\xi_{\mathrm{e}} \neq 0$ if $\theta_{\mathrm{e}} \neq 0$. It is easy to see that if the critical temperature $T_{\mathrm{c}}$ is lower than the ordering temperature $T_{\mathrm{o}}$ the symmetry of $g\left(T_{\mathrm{c}}, \xi, \theta\right)$ ensures the existence of another phase $\left(\xi_{\mathrm{e}}^{\prime}=-\xi_{\mathrm{e}}, \theta_{\mathrm{e}}^{\prime}=-y \theta_{\mathrm{e}}\right)$ with equal free energy $g\left(T_{\mathrm{c}}, \xi_{\mathrm{e}}^{\prime}, \theta_{\mathrm{e}}^{\prime}\right)=g\left(T_{\mathrm{c}}, \xi_{\mathrm{e}}, \theta_{\mathrm{e}}\right)$. When $T \neq T_{\mathrm{c}}$ the term linear in $\xi$ and $T-T_{\mathrm{c}}$ reappears in $g(T, \xi, \theta)$ and the degeneracy of the two minima is lifted. Therefore in the vicinity of $T_{\mathrm{c}}$ the free energy eq. (5) has two minima which are degenerate at $T=T_{\mathrm{c}}$. This is precisely the definition of a first-order phase transition [22]. At $T_{\mathrm{c}}$ the ordered phase with lower content of high-spin isomer $\left(\xi_{e}^{\prime}=-\xi_{e}<0\right)$ becomes less stable than the ordered phase with higher content of high-spin isomer $\left(\xi_{c}>0\right)$. The two order parameters experience the discontinuous change from $-\xi_{\mathrm{e}}$ to $\xi_{\mathrm{e}}$ and from $-y_{\mathrm{e}} \theta_{\mathrm{e}}$ to $\theta_{\mathrm{e}}$, respectively, which can be accompanied by the hysteresis effects characteristic for first-order transitions.

\section{Conclusion}

The physical picture of the spin transitions in molecular crystals can finally be presented as follows. The low- and high-spin isomers of a transition metal complex form a solid solution. The interaction between the molecules undergoing the transition does not favor the formation of pairs of isomers with the same spin. On the contrary, the intermolecular in- 
teraction is anticooperative using the terminology proposed in ref. [20]. The solid solution of the spin isomers undergoes a sublattice ordering transition. Below $T_{\mathrm{o}}$ there exist two ordered phases having different equilibrium fractions of the high-spin isomer. Below $T_{c}$ the ordered phase with the lower content of the high-spin isomer is more stable; above $T_{\mathrm{c}}$ that with the higher content of the high-spin isomer is more stable. The spin transition observed at $T_{\mathrm{c}}$ is the first-order transition between these two phases. It always takes place when the critical temperature $T_{\mathrm{c}}$ controlled by the molecular transition parameters $\Delta h$ and $\Delta s$ is lower than the ordering temperature $T_{0}$ which is controlled by the intermolecular "anticooperative" interaction.

\section{Acknowledgement}

This work has been performed during the visit of the author to the Laboratoire de Chimie Inorganique (URA CNRS 420), Orsay, whose hospitality is gratefully acknowledged as is the grant from the French Ministry of Research and Space which made this stay possible. The author is thankful to the referee for the instructive comments. He is also thankful to Professor Olivier Kahn and Professor Jaqueline Zarembowitch for valuable discussions and communication of experimental data prior to publication. Professor $O$. Kahn carefully read the manuscript and made valuable remarks concerning the style. His kind help is gratefully acknowledged.

\section{References}

[1] E. König, G. Ritter and S. Kulshreshtha, Chem. Rev. 85 (1985) 219; C.N.R. Rao, Intern. Rev. Phys. Chem. 4 (1985) 19; E. König, Struct. Bonding 76 (1991) 51.
[2] O. Kahn, J. Kröber and C. Jay, Advan. Mater. 4 (1992) 718.

[3] C.P. Slichter and H.G. Drickamer, J. Chem. Phys. 56 (1972) 2142.

[4] H.G. Drickamer and C.W. Frank, Electronic transitions and the high pressure chemistry and physics of solids (Chapman and Hall, London, 1973).

[5] M. Sorai and S. Seki, J. Phys. Chem. Solids 35 (1974) 555.

[6] 1. Prigogine, The molecular theory of solutions (Interscience, New York, 1957).

[7]E.A. Guggenheim, Mixtures (Clarendon Press, Oxford, 1952).

[8] O.K. Rice, Statistical mechanics, thermodynamics, and kinetics (Freeman, San Francisco, 1967).

[9] H. Bolvin and O. Kahn, to be published.

[10] T. Kambara, J. Chem. Phys. 70 (1979) 4199.

[11 ] T. Kambara, J. Chem. Phys. 74 (1981) 4557; N. Sasaki and T. Kambara, J. Chem. Phys. 74 (1981) 3472.

[12] S. Ramesesha, T.V. Ramakrishnan and C.N.R. Rao, J. Phys. C 12 (1979) 1307.

[13] H. Spiering, E. Meisner, H. Köppen, E.W. Müller and P. Gütlich, Chem. Phys. 68 (1982) 65.

[ 14 ] C.P. Köhler, R. Jakobi, E. Meissner, L. Wiehi, H. Spiering and P. Gütlich, J. Phys. Chem. Sol. 51 (1990) 239.

[15] H. Spiering and N. Willenbacher, J. Phys. Cond. Matter 1 (1989) 10089.

[16] P. Adler, L. Wiehl, E. Meissner, C.P. Köhler, H. Spiering and P. Gütlich, J. Phys. Chem. Sol. 48 (1987) 517.

[17] P. Gütlich, H. Köppen, R. Link and H.G. Steinhäuser, J. Chem. Phys. 70 (1979) 3977.

[18] L.D. Landau and E.M. Lifshits, Théorie de l'élasticité (Mir, Moscow, 1990).

[19] B. Gallois, J.-A. Real, C. Hauw and J. Zarembowitch, Inorg. Chem. 29 (1990) 1152.

[20] J.-A. Real, H. Bolvin, A. Bousseksou, A. Dworkin, O. Kahn, F. Varret and J. Zarembowitch, J. Am. Chem. Soc. 114 (1992) 4650.

[21] G.M. Bell and D.A. Davis, Statistical mechanics of lattice models (Ellis Horwoad, Chichester, 1989).

[22] L.D. Landau and E.M. Lifshits, Physique statistique, Vol, 1 (Mir, Moscow, 1984). 